\section{Powered bone marrow biopsy procedures produce larger core specimens, with less pain, in less time than with standard manual devices}

\author{
Larry J. Miller, ${ }^{1}$ Thomas E. Philbeck, ${ }^{1}$ \\ Diana F. Montez, ${ }^{1}$ Tatiana A. Puga, ${ }^{1}$ \\ Kim E. Brodie, ${ }^{1}$ Stephen C. Cohen, ${ }^{2}$ \\ Cathy Spadaccini, ${ }^{3}$ Ronan Swords, ${ }^{4}$ \\ Andrew J. Brenner ${ }^{4}$
}

'Vidacare Corporation, Shavano Park, TX;

2 Medical Therapy \& Research, San

Antonio, TX; ${ }^{3}$ Ameripath, San Antonio, TX; ${ }^{4}$ University of Texas Health Science Center at San Antonio, San Antonio, TX USA

\section{Abstract}

Bone marrow sampling remains essential in the evaluation of hematopoietic and many non-hematopoietic disorders. One common limitation to these procedures is the discomfort experienced by patients. To address whether a Powered biopsy system could reduce discomfort while providing equivalent or better results, we performed a randomized trial in adult volunteers. Twenty-six subjects underwent bilateral biopsies with each device. Core samples were obtained in $66.7 \%$ of Manual insertions; $100 \%$ of Powered insertions $(\mathrm{P}=0.002)$. Initial mean biopsy core lengths were $11.1 \pm 4.5 \mathrm{~mm}$ for the Manual device; $17.0 \pm 6.8 \mathrm{~mm}$ for the Powered device $(\mathrm{P}<0.005)$. Pathology assessment for the Manual device showed a mean length of $6.1 \pm 5.6 \mathrm{~mm}$, width of $1.0 \pm 0.7 \mathrm{~mm}$, and volume of $11.0 \pm 10.8 \mathrm{~mm}^{3}$. Powered device measurements were mean length of $15.3 \pm 6.1 \mathrm{~mm}$, width of $2.0 \pm 0.3 \mathrm{~mm}$, and volume of $49.1 \pm 21.5$ $\mathrm{mm}^{3}(\mathrm{P}<0.001)$. The mean time to core ejection was 86 seconds for Manual device; $47 \mathrm{sec}$ onds for the Powered device $(\mathrm{P}<0.001)$. The mean second look overall pain score was 33.3 for the Manual device; 20.9 for the Powered $(\mathrm{P}=0.039)$. We conclude that the Powered biopsy device produces superior sized specimens, with less overall pain, in less time.

\section{Introduction}

Bone marrow evaluation is essential in the diagnostic evaluation of both hematopoietic and many non-hematopoietic disorders, determining the efficacy of treatment and to monitor the recovery process in patients undergoing bone marrow transplantation or marrow-ablative chemotherapy ${ }^{1,2}$ and is also part of the staging process for newly diagnosed patients with lymphoproliferative diseases and certain non-hematopoietic malignancies. Regardless of the diagnostic value of the biopsy procedure, the pain experienced during and after the procedure makes some patients fear the procedure and/or reluctant to undergo follow-up biopsies. Attempting to mitigate the procedural pain and discomfort, some providers elect to use conscious sedation during the bone marrow procedures; but this may expose the patient to additional physical risks, increased liability to the provider, and a requirement for increased patient monitoring during and after the procedure. $^{3}$

Since 1971 the Jamshidi needle has been the device of choice for bone marrow sampling ${ }^{4}$ with no substantial advancement in marrow sampling technology thereafter. Biopsy procedures facilitated by drill-powered needles have been attempted, but with mixed results. In 1993, Ahlstrom and Astrom described a 32-patient study in which a makeshift bone biopsy system, that included a power drill, was used to obtain the bone marrow sample. Successful samples were obtained in $43 \%$ of the 37 cases. $^{5}$ More recently, Buckley et al. describe a 68 patient study in which patients underwent bone biopsy using a Black and Decker ${ }^{\mathrm{TM}}$ drill to access the iliac crest. Investigators successfully obtained diagnostic material in $80 \%$ of the cases with no major complications. ${ }^{6}$

OnControl is a battery-powered bone marrow biopsy system (Vidacare Corporation, Shavano Park, TX, USA) which allows operators to quickly and efficiently obtain both bone marrow core and aspirate (Figure 1). We recently reported the preclinical comparison of the OnControl to Manual biopsy in swine together with an uncontrolled prospective clinical evaluation for outpatient bone marrow aspiration and biopsy. These findings suggested that the Powered system was able to produce specimens of equal or greater quality faster and in a more efficient manner. ${ }^{7}$ While prospective analysis has shown a strong correlation between the duration of the procedure and the morbidity-particularly with respect to patient discomfort, ${ }^{8}$ there has not yet been a clinical study published comparing the new Powered device to traditional Manual bone marrow biopsy needles. We report on this study designed to comparatively determine if the Powered core biopsy needle has advantages over traditional Manual needles in terms of improved bone marrow sample yield, decreased pain, and procedure time.
Correspondence: Thomas E. Philbeck, 4350 Lockhill Selma Road, Shavano Park, TX 78249, USA. Tel. +1.210.375.8500 - Fax: +1.210.375-8537.

E-mail: thomas.philbeck@vidacare.com

Key words: bone marrow biopsy, core biopsy, trephine biopsy, powered biopsy.

Conflicts of interest: LJM, TEP, DFM, TAP, and KEB are employees of Vidacare Corporation, the sponsor of the study and manufacturer of one of the study devices; SCC, RS, and AJB (or their organizations) have accepted research grant funds from Vidacare Corporation, the sponsor of the study and manufacturer of one of the study devices; CS has no potential conflicts of interest.

Received for publication: 31 May 2011. Accepted for publication: 29 June 2011.

This work is licensed under a Creative Commons Attribution NonCommercial 3.0 License (CC BYNC 3.0).

(C) Copyright L.J. Miller et al., 2011

Licensee PAGEPress, Italy

Hematology Reports 2011; 3:e8

doi:10.4081/hr.2011.e8

\section{Materials and Methods}

This single-center, randomized, controlled trial was approved by IntegReview Institutional Review Board, performed in accordance with the Declaration of Helsinki and good clinical practice, and conducted in a community-based cancer clinic. After obtaining informed consent, a medical history and demographics were recorded; followed by complete blood count analysis (CBC) and a physical examination. Each subject, serving as his/her own control, received bone marrow biopsy procedures using Powered and Manual devices. The Powered device was the OnControl Bone Marrow Biopsy System (Vidacare Corporation), an FDA-cleared device consisting of a battery-powered driver and biopsy needle set. The driver resembles a small hand-held drill, and powers a single lumen needle set into the medullary cavity of the adult iliac crest. The needle set consists of two parts: an outer cannula, 11 gauge by 4 inches (102 mm) long; and a bevel-tip inner stylet - used to penetrate the cortex. The Manual device used in the study was a Jamshidi (Havel, Inc. Richmond, VA) bone marrow biopsy needle (11 gauge by 4 inches), which has a two-piece T-handle design, a trocar-tapered stylet point and a triple crown cannula tip.

Two operators (one private practice and one academic hematologist/oncologist), experienced in the use of both devices, performed the bone marrow procedures using the posteri- 
or iliac crest. The order in which the devices were used on the subject, and which side was biopsied first (right or left) was randomized. Only one attempt was allowed for each device. The aspiration component of standard bone marrow sampling was not performed for this study. There was minimal time between the two biopsy procedures; just enough for the physicians to reposition after the first procedure. Five to $7 \mathrm{~mL}$ of $1 \%$ Lidocaine buffered with sodium bicarbonate was injected intradermally, subcutaneously and periosteally as a local anesthetic for each biopsy procedure.

To assure that the subjects were not biased towards one type of biopsy needle over the other, they were carefully screened and oriented. They were told that the purpose of the trial was to see if there was a difference in the intensity of pain experienced between the two types of needles. They did not know, nor were they told, if either of the needle types was preferred by the investigators. To minimize the noise caused by the Powered device, and to decrease the potential for the noise to compromise the blinding of procedure order, noise-cancelling headphones were placed on the subjects during each procedure. To further ensure that noise was not a clue for the subject, a Powered device was activated during the Manual procedure as well. The time, in seconds, from contact of the needle with skin to sample acquisition was recorded. Pain was measured for intensity using a $100 \mathrm{~mm}$ Visual Analog Scale (VAS), with scores ranging from 0 to 100 , and higher scores indicating more pain. Participants indicated their pain level by placing a mark on a $100 \mathrm{~mm}$ line at the instant of cortical penetration, biopsy core acquisition, and needle removal. An overall procedure pain level was also recorded immediately following each needle removal. It was recognized that since these volunteers had never experienced bone marrow sampling procedures, the pain scores for the first procedure might be skewed. Therefore, following the second procedure, subjects were given the opportunity to change the overall pain score indicated for the first procedure. VAS pain scores were also recorded 30 minutes, 24 hours and 48 hours after the procedure. Subjects were also queried concerning complications at those time points, with a final complication evaluation at 7 days post procedure. Immediately following the procedures, core biopsy samples were measured and submitted to a blinded pathologist for quantitative and qualitative analysis.

Statistical testing was conducted using SPSS for Window 17.0 (SPSS, Inc. Chicago, IL, USA). Continuous parameters were summarized and compared between groups using a 2-sample ttest. Categorical parameters were summarized as proportions and compared using Fisher's Exact test. Kaplan-Meier survival analysis compared the bone marrow biopsy procedure time. A priori significance level was set at 0.05 .

\section{Results}

There were 26 subjects consented and randomized into the study. One subject was disqualified because the biopsy needle insertions sites were not adequately anesthetized before the start of procedures, and the subject did not inform investigators of the inadequacy until biopsy procedures were completed. The subject subsequently experienced severe pain bilaterally and required multiple doses of narcotic analgesia and repeated visits to medical facilities for pain relief. Another subject was obese and, after subcutaneous insertion, the first needle was not long enough to penetrate the iliac crest cortex to complete the procedure. The decision was made to not make a second attempt and the subject was disqualified. Of the 24 evaluable subjects, there were 24 Manual insertions and 24 Powered insertions. For those insertions, biopsy core samples were obtained in $66.7 \%$ of the Manual insertions and $100 \%$ of Powered insertions - a significant difference $(\mathrm{P}=0.002)$. The mean time to core acquisition was 85.7 seconds for Manual device and 46.5 seconds for the Powered device, a significant difference $(\mathrm{P}<0.001)$. Using the second look overall pain score (for those that opted to change their score), the mean score for the Manual device was 33.3 and 20.9 for the Powered - a significant difference $(\mathrm{P}=0.039)$. There was no statistical or clinical difference in other pain scores between the two devices. The initial mean biopsy core length was $11.1 \pm 4.5 \mathrm{~mm}$ for the Manual device and $17.0 \pm 6.8 \mathrm{~mm}$ for the Powered device; a statistical difference $(\mathrm{P}<0.005)$. After fixation and processing, pathology assessment for the Manual device showed a mean length of $6.1 \pm 5.6 \mathrm{~mm}$, width of $1.0 \pm 0.7 \mathrm{~mm}$, and volume of $11.0 \pm 10.8 \mathrm{~mm}^{3}$. Measurements for the Powered device were mean length of $15.3 \pm 6.1$ $\mathrm{mm}$, width of $2.0 \pm 0.3 \mathrm{~mm}$, and volume of $49.1 \pm 21.5 \mathrm{~mm}^{3}$. All differences were statistically significant with $\mathrm{P}<0.001$. For overall quality, 33.3\% of Manual samples and $79.2 \%$ of Powered samples were graded adequate - a statistical difference $(\mathrm{P}=0.002)$. See Table 1.

Between operators, there was a difference for mean time to core acquisition (75.5 seconds vs 48.4 seconds, $\mathrm{P}=0.003$ ), mean biopsy core length (16.7 mm vs $12.0 \mathrm{~mm}, \mathrm{P}=0.027)$, and mean needle insertion VAS (19.4 vs 33.6,

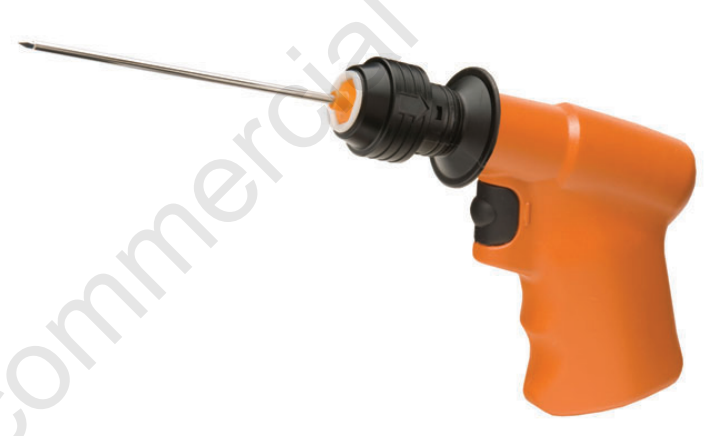

Table 1. Results by device. Results are compared with regard to device used for the procedure.

\begin{tabular}{lccc}
\hline Variable & $\begin{array}{c}\text { Manual } \\
(\mathrm{n}=24)\end{array}$ & $\begin{array}{c}\text { Powered } \\
(\mathrm{n}=24)\end{array}$ & $\mathrm{P}$ \\
Successful core acquisition, first attempt (\%) & 66.7 & 100.0 & $0.002^{*}$ \\
Mean time to core acquisition (seconds) & $85.7 \pm 31.0$ & $46.5 \pm 15.8$ & $0.000^{*}$ \\
\hline Mean biopsy core length (mm) per acquired specimen & $11.1 \pm 4.5$ & $17.0 \pm 6.8$ & $0.004^{*}$ \\
Mean VAS needle insertion (0-100) & $28.9 \pm 24.4$ & $20.5 \pm 17.3$ & 0.177 \\
\hline Mean VAS biopsy acquisition (0-100) & $36.8 \pm 20.9$ & $38.0 \pm 18.3$ & 0.134 \\
Mean VAS needle removal (0-100) & $28.8 \pm 26.6$ & $27.0 \pm 21.0$ & 0.792 \\
\hline Mean VAS overall (0-100) & $36.1 \pm 24.4$ & $23.9 \pm 16.9$ & 0.051 \\
Mean VAS overall after 2nd look adjustment (0-100) & $33.3 \pm 23.9$ & $20.9 \pm 15.6$ & $0.039^{*}$ \\
\hline Mean VAS 30 minutes post procedure (0-100) & $7.3 \pm 11.4$ & $4.4 \pm 8.5$ & 0.328 \\
Mean VAS 24 hours post procedure (0-100) & $5.8 \pm 8.5$ & $9.1 \pm 13.8$ & 0.325 \\
\hline Mean VAS 48 hours post procedure (0-100) & $1.2 \pm 1.9$ & $3.4 \pm 8.0$ & 0.201 \\
Pathology biopsy core length (mm) & $6.1 \pm 5.6$ & $15.3 \pm 6.1$ & $<0.001^{*}$ \\
\hline Pathology biopsy core width (mm) & $1.0 \pm 0.7$ & $2.0 \pm 0.3$ & $<0.001^{*}$ \\
\hline Pathology biopsy core volume (mm $\left.{ }^{3}\right)$ & $11.0 \pm 10.8$ & $49.1 \pm 21.5$ & $<0.001^{*}$ \\
\hline Pathology-graded adequate biopsy core (\%) & 33.3 & 79.2 & $0.002^{*}$ \\
\hline *Denotes statistical significance; VAS, Visual Analog Scale. & & &
\end{tabular}

Figure 1. The powered bone marrow biopsy system. The power driver and biopsy needle components of the OnControl powered bone marrow sampling system. 
$\mathrm{P}=0.024)$. Between operators, there was no statistical difference in other VAS pain scores, ability to capture a core specimen or proportion of core biopsy specimens graded adequate by pathology (See Table 2).

The only complication noted during the study was intense and extended pain experienced by one subject, which took nearly 4 weeks to subside.

\section{Discussion}

In a 2009 article describing a 48-patient study that assessed pain during biopsy procedures, Ruegg and associates reported mean biopsy needle insertion pain to be 38.5 when patients were anesthetized with buffered lidocaine. ${ }^{3}$ In our study, in which buffered lidocaine was also used, subjects rated their pain at 38.0 on average with the Powered device. When given the opportunity to change the score for the first procedure, the mean pain score for the Powered insertions was reduced to 20.9 .

The procedure time (needle-to-skin contact to core biopsy sample acquisition) was substantially shorter when using the Powered device. Indeed, in Kuball's 2004 publication describing 263 patients undergoing bone marrow procedures, investigators reported that the duration of the procedure, which averaged 7 minutes, was identified as the sole independent predictive factor for patients' pain intensity. ${ }^{8}$ The investigators did not define how the duration of needle insertion was measured, but the finding was quantitatively and qualitatively substantiated in our study. This was particularly true for patients with harder bones in which more time and effort is required for cortical penetration. In our study, 154 seconds were required for one subject using the Manual needle, compared to 70 seconds using the Powered device. For this subject, the Manual procedure resulted in a bent needle (Figure 2) and no biopsy core sample.

While clinicians strive to conduct bone marrow sampling procedures with as little pain and discomfort to the patient as possible, the ultimate goal of the procedure is the acquisition of a specimen suitable for diagnosis by pathologists. Inadequate specimens have a significant impact on clinicians' ability to treat patients whose treatment depend an accurate diagnosis. In a 767 patient study, Bishop et al. reported that only $42 \%$ of their biopsy specimens were adequate for accurate diagnosis. ${ }^{9}$ An adequate sample must be of sufficient size and relatively free of crush artifact and trabecular distortion. Greater amounts of tissue increase the chances of identifying focal lesions. The superior core specimens acquired using the Powered device is the key finding of this study. Aside from a 100\% first attempt core capture rate compared to $67 \%$ for the Manual device, the Powered device delivered core specimens that were $53 \%$ longer, and contained $346 \%$ more volume (Figure 3).

A limitation of our study was the use of healthy volunteer subjects, rather than actual patients. We found it would be difficult and time-consuming for clinicians to accrue a satisfactory number of patients requiring bi-lateral procedures. A second reason is because pain as a study outcome is generally problematic in actual patients due to the great amount of variability, bias and subjectivity. These confounding factors are multiplied with cancer patients who often have underlying pain and an altered perception of pain. Another limitation was the decision not to collect a bone marrow aspirate - a normal component of bone marrow sampling procedures. For many patients, the negative pressure within the medullary space during the syringe aspiration causes the worst pain of the procedure, and the intensity of pain is not a function of needle-type. For this rea-

Table 2. Results by operator. Results are compared with regard to operator performing the procedure.

\begin{tabular}{lccc}
\hline Variable & $\begin{array}{c}\text { Operator A } \\
(\mathrm{n}=30)\end{array}$ & $\begin{array}{c}\text { Operator B } \\
(\mathrm{n}=20)\end{array}$ & P \\
Successful core acquisition, first attempt (\%) & 76.7 & 94.4 & 0.113 \\
Mean time to core acquisition (seconds) & $75.5 \pm 33.2$ & $48.4 \pm 17.6$ & $0.003^{*}$ \\
\hline Mean biopsy core length (mm) per acquired specimen & $16.6 \pm 6.9$ & $12.0 \pm 5.2$ & $0.027^{*}$ \\
Mean VAS needle insertion both needles (0-100) & $19.4 \pm 17.4$ & $33.6 \pm 24.7$ & $0.024^{*}$ \\
\hline Mean VAS biopsy acquisition both needles (0-100) & $28.6 \pm 18.9$ & $38.7 \pm 20.6$ & 0.090 \\
Mean VAS needle removal both needles (0-100) & $26.3 \pm 23.5$ & $30.6 \pm 24.7$ & 0.550 \\
\hline Mean VAS overall both needles (0-100) & $24.8 \pm 20.1$ & $34.0 \pm 22.5$ & 0.327 \\
Mean VAS overall after 2nd look adjustment both needles (0-100) & $27.6 \pm 21.2$ & $30.9 \pm 22.3$ & 0.332 \\
\hline Mean VAS 30 minutes post procedure both needles (0-100) & $5.3 \pm 9.9$ & $6.8 \pm 10.6$ & 0.607 \\
Mean VAS 24 hours post procedure both needles (0-100) & $9.0 \pm 12.8$ & $5.0 \pm 8.9$ & 0.247 \\
\hline Mean VAS 48 hours post procedure both needles (0-100) & $2.6 \pm 7.2$ & $1.8 \pm 2.6$ & 0.641 \\
Pathology biopsy core length (mm) & $11.0 \pm 8.1$ & $10.2 \pm 6.3$ & 0.711 \\
\hline Pathology biopsy core width (mm) & $1.4 \pm 0.8$ & $1.7 \pm 0.6$ & 0.187 \\
Pathology biopsy core volume (mm $\left.{ }^{3}\right)$ & $31.5 \pm 27.8$ & $27.7 \pm 21.8$ & 0.629 \\
\hline Pathology-graded adequate biopsy core (\%) & 60.0 & 50.0 & 0.104 \\
\hline
\end{tabular}

*Denotes statistical significance; VAS, Visual Analog Scale.

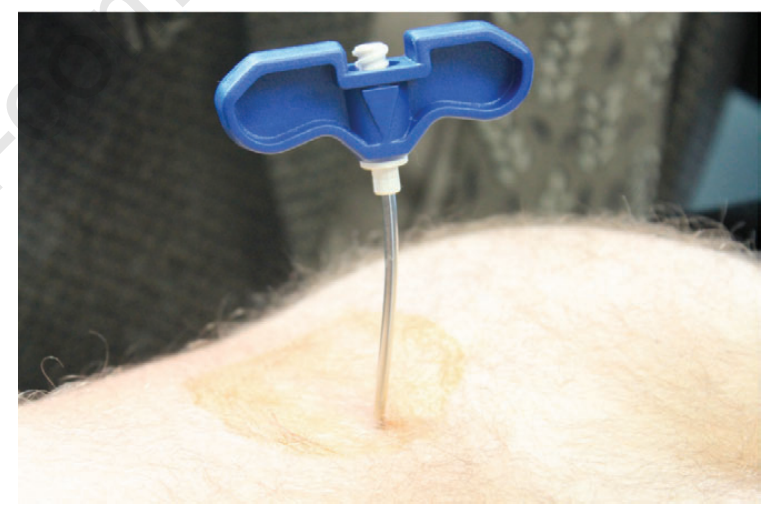

Figure 2. Manual bone marrow biopsy needle. This shows the condition of the manual needle following insertion into a patient with hard bones.

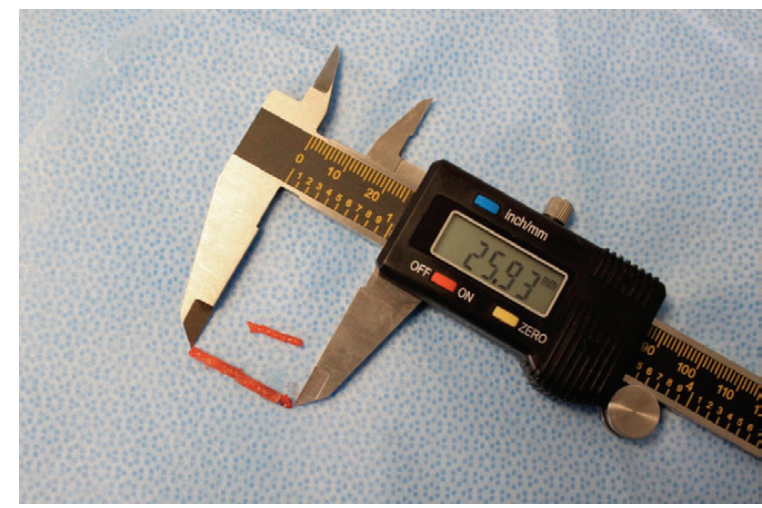

Figure 3. Biopsy core specimens. These are typical examples of bone marrow core specimens obtained following bilateral bone marrow biopsy procedures. Upper specimen is from Manual device and lower specimen is from Powered device. 
son, we opted to eliminate the aspiration component. One other limitation was our failure to standardize the amount of time between the local anesthetic infiltration and the biopsy procedure. An additional limitation was failure to capture total pain (e.g. intensity of the pain times the duration of the pain). While the difference in the intensity of the pain did not reach statistical significance in several of the parameters tested, there is little doubt that the duration of the pain was substantially longer in the Manual arm of the study. Several subjects stated that they would much rather have the Powered needle because the procedure was faster.

\section{Conclusions}

Results suggest the superior size and overall quality of core specimens delivered by the Powered device provide more material for pathological evaluation of hematopoietic and oncological disorders. The Powered device was significantly faster in obtaining a biopsy than the Manual device. The capture rate in obtaining a satisfactory sample on first attempt was much higher with the Powered device, negating the necessity of conducting a second biopsy. While the Powered bone marrow biopsy showed a trend toward decreased pain overall at the time of the procedures, use of the Powered bone marrow biopsy device did significantly decreased overall procedure pain when subjects considered the two procedures retrospectively.

\section{References}

1. Snover DC. Biopsy interpretation in bone marrow transplantation. Pathol Annu 1989;24:64-101.

2. Islam A, Henderson ES. Value of long-core biopsy in the detection of discrete bone marrow lesions. Histopathology 1988;12: 641-8.

3. Ruegg TA, Curran CR, Lamb T. Use of buffered Lidocaine in bone marrow biopsies: a RCT: theoretical framework. Oncol
Nurs Forum 2009;36:52-60.

4. Jamshidi K, Swaim WR. Bone marrow biopsy with unaltered architecture: a new biopsy device. J Lab Clin Med 1971:77;33542.

5. Ahlstrom KH, Astrom KGO. CT-guided bone biopsy performed by means of a coaxial biopsy system with an eccentric drill. Radiology 1993;188:549-52.

6. Buckley 0, Benfayed W, Geoghegan T, et al. CT-guided bone biopsy: Initial experience with a commercially available hand held Black and Decker ${ }^{\mathrm{TM}}$ drill. European Journal of Radiology 2007;61:176-80.

7. Swords RT, Kelly KR, Cohen S, Miller LJ. Rotary powered device for bone marrow aspiration and biopsy yields excellent specimens quickly and efficiently. J Clin Pathol 2010;63:562-5.

8. Kuball J, Schuz J, Gamm H, Weber M. Bone marrow punctures and pain. Acute Pain 2004;6:9-14.

9. Bishop PW, McNally K, Harris M. Audit of bone marrow trephines. J Clin Pathol 1992;45:1105-8. 